\title{
Noniterative Phase Retrieval from a Single Diffraction Intensity Pattern by Use of an Aperture Array
}

\begin{abstract}
Nobuharu Nakajima
Faculty of Engineering, Shizuoka University, 3-5-1 Johoku, Hamamatsu, 432-8561, Japan

(Received 19 September 2006; revised manuscript received 31 January 2007; published 31 May 2007)

A noniterative method for retrieving the phase of a wave field from a diffraction intensity measurement in a coherent wave field is proposed. In this method, the phase can be calculated from analytic equations based on the properties of entire functions by use of Fourier transforms. This method requires only a single measurement of the intensity of a diffracted wave through an array filter of rectangular apertures and then does not need to use any lens systems and coherent reference waves. Therefore, it provides a potentially useful means for coherent imaging in a wide class of wave fields such as optical, x ray, electron, and atomic waves.
\end{abstract}

DOI: 10.1103/PhysRevLett.98.223901

PACS numbers: $42.30 . \mathrm{Rx}, 42.30 . \mathrm{Kq}, 42.30 . \mathrm{Wb}$

A lensless coherent imaging of an object from its diffraction intensity is very attractive for various applications because the resolution of such a method is not limited by the aberration and the finite extent of a lens. To achieve this, we have to retrieve the phase of the diffraction field, which is lost on an intensity recording. One approach to such lensless imaging is holography. However, for highfrequency waves, such as $\mathrm{x}$ rays and electron waves, a coherent reference source of a very small size, by which the spatial resolution is limited, is necessary for offsetreference holography [1], and also there exists the socalled twin-image problem in Gabor's inline holography. Thus, nonholographic methods for the object reconstruction from diffraction intensities have been recently developed [2] when it is inconvenient to generate a coherent reference wave. One well-known approach is the use of an iterative method that was proposed by Fienup [3] as a modification of the original Gerchberg-Saxton algorithm [4]. For example, object reconstruction by use of the iterative method has been demonstrated in x-ray phaseretrieval experiments for noncrystalline samples [5] and the coherent electron imaging of a carbon nanotube from its diffraction intensity [6]. The use of the iterative method, however, is accompanied by convergence problems, and hence the method sometimes stagnates in a local minimum solution different from a true one, especially in the reconstruction of complex-amplitude objects [7].

On the other hand, there have been some studies of noniterative (analytic) and nonholographic phase-retrieval methods. For example, phase recovery by solution of the transport-of-intensity equation has been recently developed $[8,9]$ and applied to phase-retrieval in electron microscopy [10], x-ray imaging [11], and so forth. While Fienup's iterative method needs only a diffraction pattern, all of the noniterative and nonholographic methods proposed for practical use so far require more than two intensity measurements, except for the cases with special conditions such as a nonabsorbing object [11] and a monomorphous object [12]. The object reconstruction from a single diffraction intensity enables us to observe an object in real time, and also it is significant for the reduction of sample damage by an illuminating radiation such as an $\mathrm{x}$-ray or an electron wave.

In this Letter, a noniterative method based on the properties of entire functions $[13,14]$ is proposed, in which phase recovery is possible from a single diffraction intensity distribution of a wave field transmitted through an array filter of rectangular apertures. To the best of my knowledge, this is the first noniterative and nonholographic phase-retrieval method using a single intensity measurement for practical use without such special conditions.

Figure 1 shows a schematic diagram of the present phase-retrieval method. We assume that an object of complex-amplitude transmittance $f(u, v)$ (which is assumed to be of finite extent $\sigma$ ) in the object plane is illuminated by a coherent monochromatic plane wave of wavelength $\lambda$. In the far-field plane at a distance of $z$ downstream of the object with coordinates $x$ and $y$, an array filter consisting of rectangular apertures is inserted to take a correlation of the rectangular function and the field distribution of the diffracted wave from the object. We here consider the case of a $N \times M$ array of square apertures of each width $w$ distributed over a Cartesian grid of period $d$.

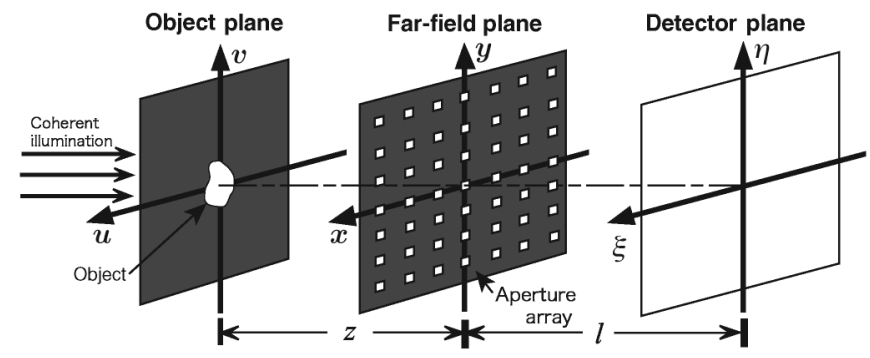

FIG. 1. Schematic diagram of the measurement system for the object reconstruction by the phase-retrieval method. The object function is reconstructed from a single intensity distribution of a diffracted wave through an array of rectangular apertures. 
Using the Fraunhofer and the Fresnel diffraction integrals, we can obtain the amplitude distribution in the detector plane at a distance of $l$ downstream of the array filter as

$$
\begin{aligned}
K(\xi, \eta)= & \sum_{n=-N / 2}^{N / 2-1} \sum_{m=-M / 2}^{M / 2-1} \iint_{-\infty}^{\infty} F(x, y) \exp \left[i \frac{\pi}{\lambda z}\left(x^{2}+y^{2}\right)\right] \\
& \times R\left(x-x_{n}, y-y_{m}\right) \\
& \times \exp \left\{i \frac{\pi}{\lambda l}\left[(x-\xi)^{2}+(y-\eta)^{2}\right]\right\} d x d y
\end{aligned}
$$

where $F(x, y)$ is the complex-amplitude distribution in the far-field plane, which is given by

$$
F(x, y)=\iint_{\sigma} f(u, v) \exp \left[-i \frac{2 \pi}{\lambda z}(x u+y v)\right] d u d v,
$$

and $R\left(x-x_{n}, y-y_{n}\right)$ denotes the amplitude transmittance of a square aperture being at the position of the coordinates $\left(x_{n}, y_{m}\right)=(n d, m d), \quad[R(x, y)=1.0$ for $-w / 2 \leq x \leq$ $w / 2,-w / 2 \leq y \leq w / 2$ and $R(x, y)=0$ otherwise]. In Eqs. (1) and (2), unimportant multiplicative constants associated with the diffraction integrals are ignored. In order to combine two functions of the quadratic phases in the integral of Eq. (1), we substitute the coordinates $\xi_{n}=$ $x_{n}(1+l / z)$ and $\eta_{m}=y_{m}(1+l / z)$ into the coordinates $\xi$ and $\eta$ in Eq. (1). Then Eq. (1) is rewritten as

$$
\begin{aligned}
K\left(\xi_{n}, \eta_{m}\right)= & \sum_{n=-N / 2}^{N / 2-1} \sum_{m=-M / 2}^{M / 2-1} q \iint_{-\infty}^{\infty} F(x, y) R\left(x-x_{n}, y-y_{m}\right) \\
& \times \exp \left\{i \frac{\pi}{\lambda l}\left(1+\frac{l}{z}\right)\left[\left(x-x_{n}\right)^{2}+\left(y-y_{m}\right)^{2}\right]\right\} d x d y,
\end{aligned}
$$

where $q=\exp \left[i \pi(1+l / z)\left(x_{n}^{2}+y_{m}^{2}\right) / \lambda z\right]$. Here we assume that the Fresnel diffraction pattern of each small aperture can be regarded as the Fraunhofer diffraction one in the detector plane, although the period $d$ of the aperture array is large enough to produce the periodic pattern of the geometrical optics description of the array in the region of Fresnel diffraction. Therefore, the quadratic phase factor in Eq. (3) is approximately unity over the aperture function $R\left(x-x_{n}, y-y_{m}\right)$. In addition, we assume that the diffraction pattern of each square aperture in the detector plane is isolated approximately from those of the adjoining square apertures. Then the observable intensity of Eq. (3) is given by

$$
\left|K\left(\xi_{n}, \eta_{m}\right)\right|^{2} \cong\left|\iint_{-\infty}^{\infty} F(x, y) R\left(x-x_{n}, y-y_{m}\right) d x d y\right|^{2} .
$$

A sufficient condition for the isolation of the diffraction patterns of the neighboring apertures would be that the main width of the Fraunhofer pattern of each aperture is smaller than the period of the array pattern projected on the detector plane. Substituting Eq. (2) into Eq. (1) and calcu- lating the resultant equation under the Fraunhofer approximation, we obtain a convolution of the object with the Fourier transform (i.e., a sinc function) of each square aperture. Then the rough width $H$ of the Fraunhofer pattern from a square aperture can be estimated by $H \cong 2 \lambda l / w+$ $l \sigma_{j} / z,(j=u, v)$ where $2 \lambda l / w$ denotes the main lobe between the first two zeros of the sinc function, and $\sigma_{u}$ and $\sigma_{v}$ denote the extent of the object in the direction of $u$ and $v$, respectively. On the other hand, the period of the Fresnel diffraction pattern of the array in the detector plane is given from the geometrical optics prediction as $d(1+$ $l / z$ ), where the term $l / z$ represents the effect of the extension generated by the quadratic phase term $\exp \left[i \pi\left(x^{2}+\right.\right.$ $\left.\left.y^{2}\right) / \lambda z\right]$ in Eq. (1). Consequently, if the condition $H<$ $d(1+l / z)$ is satisfied in the measurement system, then we may regard Eq. (4) as the intensity distribution at the coordinates $\left(\xi_{n}, \eta_{m}\right)$ in the detector plane.

Equation (4) indicates that the intensities at the points of coordinates $\left(\xi_{n}, \eta_{m}\right)(n=-N / 2, \ldots, 0, \ldots, N / 2-1$, and, $m=-M / 2, \ldots, 0, \ldots, M / 2-1)$ in the detector plane correspond to the intensity distribution of the correlation integral of the aperture function $R(x, y)$ and the Fourier transform $F(x, y)$ of the object function in Eq. (2). By the sampling theorem, the intensity distribution of the correlation integral can be encoded into the discrete data of Eq. (4) when the period $d$ of the array is smaller than $\lambda z / 2 \sigma_{j}(j=u, v)$. The parameters $d$ and $z$, which fulfill the condition for encoding, can be determined from the roughly estimated extent of an object by another measurement. Under this condition, we may regard the arguments $x_{n}$ and $y_{m}$ in functions as continuous variables.

Using the same way as in Eqs. (3) and (4), we can obtain the intensity distributions at another point of coordinates $\left(\xi_{n}+\tau, \eta_{m}\right)$ and $\left(\xi_{n}, \eta_{m}+\tau\right)$ (where $\tau$ is a known constant):

$$
\begin{aligned}
\left|K\left(\xi_{n}+\tau, \eta_{m}\right)\right|^{2} \cong & \mid \iint_{-\infty}^{\infty} F(x, y) R\left(x-x_{n}, y-y_{m}\right) \\
& \times\left.\exp \left(-i \frac{2 \pi}{\lambda l} x \tau\right) d x d y\right|^{2},
\end{aligned}
$$

and $\left|K\left(\xi_{n}, \eta_{m}+\tau\right)\right|^{2}$ is represented by substituting $\exp (-i 2 \pi y \tau / \lambda l)$ into Eq. (5) instead of $\exp (-i 2 \pi x \tau / \lambda l)$. Then one can retrieve the twodimensional (2-D) phase of the correlation integral in Eq. (4) from $\left|K\left(\xi_{n}, \eta_{m}\right)\right|^{2}, \quad\left|K\left(\xi_{n}+\tau, \eta_{m}\right)\right|^{2}$, and $\left|K\left(\xi_{n}, \eta_{m}+\tau\right)\right|^{2}$ by using the phase-retrieval method based on the properties of entire functions.

To utilize the properties of entire functions, we assume that the extent of the inverse Fourier transform (i.e., a sinc function) of a square aperture $R(x, y)$ has an extent large enough to be approximated into a Gaussian function within the extent of the object function [15]. From studies of numerical experiments, the Gaussian approximation was found to be effective, provided that the extent of the object function was smaller than about $(1 / 2)$ of the extent between the first two zeros of the sinc function. The mea- 
surement system that satisfies this requirement can be prepared for in advance through the roughly estimated extent of an object. Thus, we approximate the inverse Fourier transform of an aperture $R(x, y)$ of width $w$ into a Gaussian function [16],

$$
\iint_{-\infty}^{\infty} R(x, y) \exp \left[i \frac{2 \pi}{\lambda z}(u x+v y)\right] d x d y=w^{2} \operatorname{sinc}\left(\frac{w}{\lambda z} u\right) \operatorname{sinc}\left(\frac{w}{\lambda z} v\right) \cong w^{2} \exp \left[-\frac{1}{6}\left(\frac{\pi w}{\lambda z}\right)^{2}\left(u^{2}+v^{2}\right)\right] .
$$

Substituting the Fourier transform of the Gaussian function instead of $R(x, y)$ into Eqs. (4) and (5), we can obtain the following expressions respectively:

$$
\begin{gathered}
\left|K\left(\xi_{n}, \eta_{m}\right)\right|^{2} \cong\left|\iint_{-\infty}^{\infty} F(x, y) G\left(x-x_{n}, y-y_{m}\right) d x d y\right|^{2}, \\
\left|K\left(\xi_{n}+\tau, \eta_{m}\right)\right|^{2} \cong b\left|\iint_{-\infty}^{\infty} F(x, y) G\left[x-\left(x_{n}-i c\right), y-y_{m}\right] d x d y\right|^{2},
\end{gathered}
$$

where $b$ and $c$ are constant values $\exp \left[-(\pi \tau w / \sqrt{3} \lambda l)^{2}\right]$ and $\pi \tau w^{2} /(6 \lambda l)$, respectively, and the function $G(x, y)$ is given by the Fourier transform of the Gaussian function in Eq. (6). The equation for $\left|K\left(\xi_{n}, \eta_{m}+\tau\right)\right|^{2}$ is represented by substituting $G\left[x-x_{n}, y-\left(y_{m}-i c\right)\right]$ into Eq. (8) instead of $G\left[x-\left(x_{n}-i c\right), y-y_{m}\right]$. As proven in the previous paper [14], two moduli along the real axis $x_{n}$ and the line $x_{n}-i c$ parallel to the real axis in the complex plane (i.e., Eqs. (7) and (8), respectively) have sufficient information for one to retrieve the phase of the correlation integral in Eq. (7). Let $M\left(x_{n}, y_{m}\right)$ and $\phi\left(x_{n}, y_{m}\right)$ be the modulus and the phase, respectively, of the correlation integral in the right hand side of Eq. (7), i.e.,

$$
\iint_{-\infty}^{\infty} F(x, y) G\left(x-x_{n}, y-y_{m}\right) d x d y=M\left(x_{n}, y_{m}\right) \exp \left[i \phi\left(x_{n}, y_{m}\right)\right]
$$

To retrieve one-dimensional (1-D) phases along lines parallel to the $x$ axis, the real variable $x_{n}$ in Eq. (9) is expanded into the complex one, $x_{n}-i c$. Then Eq. (9) is rewritten as

$$
\iint_{-\infty}^{\infty} F(x, y) G\left[x-\left(x_{n}-i c\right), y-y_{m}\right] d x d y=M\left(x_{n}-i c, y_{m}\right) \exp \left\{i\left[\phi_{R}\left(x_{n}, y_{m}\right)+i \phi_{I}\left(x_{n}, y_{m}\right)\right]\right\},
$$

where $\phi_{R}\left(x_{n}, y_{m}\right)$ and $\phi_{I}\left(x_{n}, y_{m}\right)$ denote the real and imaginary parts of the complex function $\phi\left(x_{n}-i c, y_{m}\right)=$ $\phi_{R}\left(x_{n}, y_{m}\right)+i \phi_{I}\left(x_{n}, y_{m}\right)$, respectively. Then substitution of Eq. (10) into Eq. (8) yields a relation

$$
\ln \left[\frac{\left|K\left(\xi_{n}+\tau, \eta_{m}\right)\right|}{\left|M\left(x_{n}-i c, y_{m}\right)\right|}\right]-\frac{1}{2} \ln b \cong-\phi_{I}\left(x_{n}, y_{m}\right) \text {. }
$$

The left-hand side of Eq. (11) is an obtainable function because the term $\ln b$ consists of known constants, and because the numerator in the first term is the square root of the intensity distributions $\left|K\left(\xi_{n}+\tau, \eta_{m}\right)\right|^{2}$, and the denominator in the first term can be calculated numerically from the intensity distribution $M^{2}\left(x_{n}, y_{m}\right)\left[\cong\left|K\left(\xi_{n}, \eta_{m}\right)\right|^{2}\right]$ by taking the 1-D Fourier transform of the product of the inverse Fourier transform of the modulus $M\left(x_{n}, y_{m}\right)$ $\left[\cong\left|K\left(\xi_{n}, \eta_{m}\right)\right|\right]$ for the $x_{n}$ coordinate and an exponential function $\exp (-2 \pi c u)$ in the same way as in the previous paper [15].

Thus, from Eq. (11), the 1-D phases of $\phi\left(x_{n}, y_{m}\right)$ along lines parallel to the $x$ axis in Eq. (9) can be obtained by using Fourier transforms as shown in Eqs. (10) to (16) of the previous paper [15]. The resultant equation is represented by

$$
\phi\left(x_{n}, y_{m}\right)=\mathfrak{\Im}^{-1}\left[\frac{\mathfrak{I}\left[D\left(x_{n}, y_{m}\right)\right]}{i \sinh (2 \pi c \alpha)}\right],
$$

where $D\left(x_{n}, y_{m}\right)=\ln \left[\left|K\left(\xi_{n}+\tau, \eta_{m}\right)\right| /\left|M\left(x_{n}-i c, y_{m}\right)\right|\right]-$
$(1 / 2) \ln b$, and $\Im[\cdots]$ and $\Im^{-1}[\cdots]$ denote a 1 -D Fourier transform of a function of the variable $x_{n}$ and an inverse Fourier transform of a function of the variable $\alpha$, respectively. The 1-D phases of $\phi\left(x_{n}, y_{m}\right)$ along lines parallel to the $y$ axis can also be retrieved from the intensity distributions $\left|K\left(\xi_{n}, \eta_{m}\right)\right|^{2}$ and $\left|K\left(\xi_{n}, \eta_{m}+\tau\right)\right|^{2}$ by using the same procedures as in Eqs. (9)-(12). Then the overall 2-D phase $\phi\left(x_{n}, y_{m}\right)$ of the correlation integral in Eq. (9) can be determined by addition of each phase along one line of the 1-D lines parallel to the $y$ axis to the 1-D phases along the lines parallel to the $x$ axis, because a phase distribution along one line in the $y$-axis direction can be regarded as the constant phase difference among the 1-D phases along the lines parallel to the $x$ axis. Thus, by an inverse Fourier transform of the correlation integral that consists of the measured modulus $M\left(x_{n}, y_{m}\right)\left[\cong\left|K\left(\xi_{n}, \eta_{m}\right)\right|\right.$ in Eq. (7)] and the retrieved phase $\phi\left(x_{n}, y_{m}\right)$, we can reconstruct the product of the object function and the Gaussian function in Eq. (6). Finally, the object function $f(u, v)$ can be obtained through compensation for the known Gaussian function. The errors of the Gaussian approximation bring the slight change of the object's modulus like noises do [16], and so have no direct influence on the spatial resolution. Hence, the spatial resolution is determined by about $2 \lambda z / S$ (where $S=N d$ or $M d$ is the side length of the array filter), which is the same as that of iterative methods with the same conditions. 


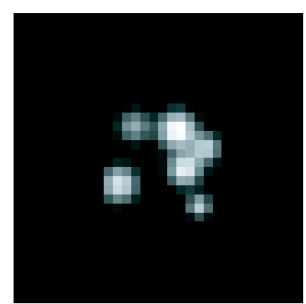

(a)

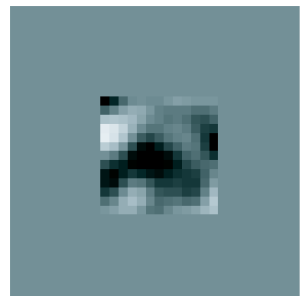

(b)

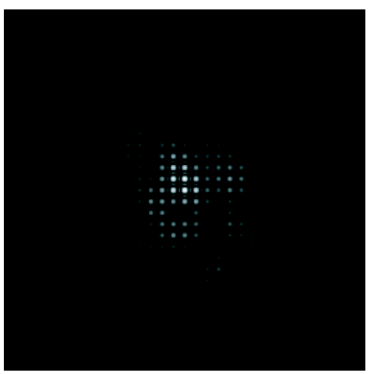

(c)

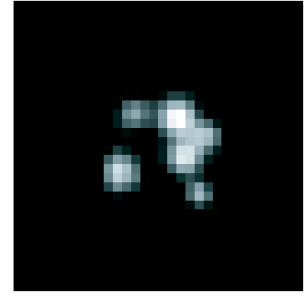

(d)

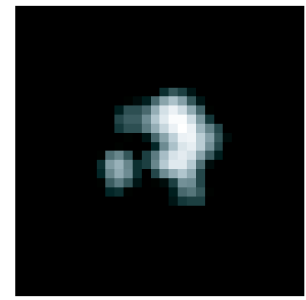

(f)

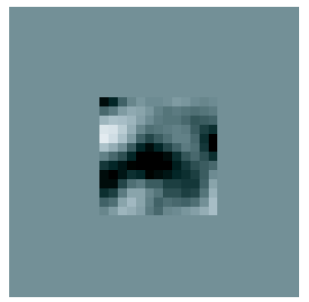

(e)

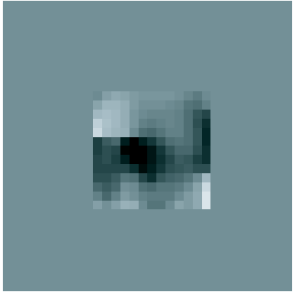

(g)
FIG. 2 (color online). Reconstruction of a complex-valued object from a single intensity distribution by the phase-retrieval method: (a) modulus and (b) phase of the original object (where the values of the phase are in the range of -2.52 to $2.51 \mathrm{rad}$ ), and (c) modulus in the detector plane of Fig. 1. (d) and (e) [or (f) and (g)] are the modulus and phase, respectively, of a reconstructed object from the noiseless (or noisy) modulus of (c).

The performance of the present method is demonstrated by an example of computer simulations for the reconstructions of complex-valued objects. We assume that the object is illuminated by a monochromatic plane wave with wavelength $0.1 \mathrm{~nm}$ (e.g., an $\mathrm{x}$ ray or an electron wave). The distances between the object and the far-field planes and between the far-field and the detector planes are assumed to be $z=100 \mathrm{~mm}$ and $l=300 \mathrm{~mm}$, respectively. In the far-field plane, we place an aperture array, which composes of a uniform square grid of interval $d=15.0 \mu \mathrm{m}$ with $32 \times 32$ square apertures of each width $w=1.88 \mu \mathrm{m}$. Figures 2(a) and 2(b) show the modulus and the phase of the original object function, where the figures are repre- sented by $32 \times 32$ points. The object is assumed to have a finite square extent, shown in the central $16 \times 16$ points of the figures, of which the physical size is assigned to $333 \times$ $333 \mathrm{~nm}$. The distribution of the phase is in the range of from -2.52 to $2.51 \mathrm{rad}$. Data processing for calculating the Fresnel diffraction integral by Fourier transforms was carried out with $1024 \times 1024$ sampling points, of which the central part $(16 \times 16$ points $)$ corresponds to the object and the rest of the sampling points was padded with zeros. Figure 2(c) shows the modulus (the square root of the intensity distribution) in the detector plane. Figures 2(d) and 2(e) [or 2(f) and 2(g)] show the modulus and the phase, respectively, of the reconstructed object from noiseless (or noisy) intensity data. The signal-to-noise ratio, which is defined by the ratio of the total intensities for signals and noises in the detector plane, in Figs. 2(f) and 2(g) becomes 661. The normalized root-mean-square error in Figs. 2(d) and 2(e) and, Figs. 2(f) and 2(g) are 0.138 and 0.609, respectively.

In this Letter, we have developed a noniterative and nonholographic phase-retrieval method that allows the object reconstruction from only a single diffraction pattern of a wave field transmitted through an aperture array. Although this method needs an array filter of apertures, it would be possible to make such a filter by using the technique of electron beam lithography.

[1] S. Eisebitt et al., Nature (London) 432, 885 (2004).

[2] K. A. Nugent, D. Paganin, and T. E. Gureyev, Phys. Today 54, No. 8, 27 (2001)

[3] J. R. Fienup, Appl. Opt. 21, 2758 (1982).

[4] R. W. Gerchberg and W. O. Saxton, Optik (Stuttgart) 35, 237 (1972).

[5] J. Miao et al., Phys. Rev. Lett. 89, 088303 (2002).

[6] J. M. Zuo et al., Science 300, 1419 (2003).

[7] J. R. Fienup, J. Opt. Soc. Am. A 4, 118 (1987).

[8] M. R. Teague, J. Opt. Soc. Am. 73, 1434 (1983).

[9] T. E. Gureyev, A. Roberts, and K. A. Nugent, J. Opt. Soc. Am. A 12, 1932 (1995).

[10] S. Bajt et al., Ultramicroscopy 83, 67 (2000).

[11] K. A. Nugent et al., Phys. Rev. Lett. 77, 2961 (1996).

[12] L. D. Turner, K. F. E. M. Domen, and R. E. Scholten, Phys. Rev. A 72, 031403(R) (2005).

[13] R. E. Burge et al., Proc. R. Soc. A 350, 191 (1976).

[14] N. Nakajima, J. Opt. Soc. Am. A 5, 257 (1988).

[15] N. Nakajima, Appl. Opt. 44, 6228 (2005).

[16] The Maclaurin expansion of the sinc and the Gaussian functions are given by $\operatorname{sinc}(x)=\sin (\pi x) / \pi x=$ $1-(\pi x)^{2} / 6+(\pi x)^{4} / 120-\cdots$, and $\exp \left[-(\pi x)^{2} / 6\right]=$ $1-(\pi x)^{2} / 6+(\pi x)^{4} / 72+\cdots$, respectively. The normalized errors $\left|\operatorname{sinc}(x)-\exp \left[-(\pi x)^{2} / 6\right]\right| / \operatorname{sinc}(x)$ become smaller than about $4 \%$ within the half extent between the first two zeros of $\operatorname{sinc}(x)$. From the computer simulations, the effect of the errors on the object reconstruction was estimated at less than several percentages of the average modulus of an object, which is regarded as the rough limit of dynamic resolution. 